\title{
Nonlinear multivariate modeling of strand mechanical properties with near-infrared spectroscopy
}

\author{
by Brian K. Via ${ }^{1}, 2,{ }^{*}$ and W. Jiang ${ }^{1,2}$
}

\begin{abstract}
The purpose of this research was to determine if nonlinear calibrations of near-infrared spectra could improve the prediction of strand mechanical properties. Strands similar in dimension to strands utilized in an oriented strand board composite process were prepared and tested for mechanical properties in three-point bending and then calibrated to near-infrared reflectance spectroscopy. It was found that an additional $7 \%$ to $16 \%$ of the variation in mechanical properties could be accounted for when second- and third-order terms were applied. Interpretation of models identified the magnitude of importance that various wood polymers play on mechanical properties and this interpretation was validated through wet chemistry. This work is significant because it demonstrates the potential of using near-infrared spectroscopy to monitor shifts in strand mechanical properties prior to wood composite manufacture and it helps to provide the fundamental relationship between near-infrared models, wood chemistry, and the prediction of mechanical properties.
\end{abstract}

Keywords: near-infrared, NIR, spectroscopy, strand, flake, biomass, composite, chemistry, strength, stiffness, elasticity

\section{RÉSUMÉ}

Le but de cette recherche est de déterminer si létalonnage non-linéaire de spectres proche infrarouge peut améliorer la prédiction des propriétés mécaniques des copeaux de bois. Des copeaux de dimensions similaires à ceux utilisés dans le processus de fabrication des panneaux à copeaux orientés ont été préparés et leurs propriétés mécaniques testées en flexion à trois points puis étalonnées au moyen de la spectrométrie proche infrarouge en réflectance. Il a été trouvé que $7 \%$ à $16 \%$ de variation additionnelle des propriétés mécaniques pouvaient être prises en compte en appliquant des termes du deuxième et troisième degré. L'interprétation des modèles a permis d'identifier l'importance que différents polymères du bois jouent sur les propriétés mécaniques et cette interprétation a été validée au moyen de tests chimiques. Ce travail est important parce quil démontre le potentiel de l'utilisation de la spectrométrie proche infrarouge pour contrôler les changements dans les propriétés mécaniques des copeaux avant la fabrication des panneaux et aide à fournir une relation fondamentale entre les modèles proche infrarouge, la chimie du bois et la prédiction des propriétés mécaniques.

Mots clés : proche infrarouge, PIR, spectroscopie, copeaux, particules, biomasse, composé, chimie, résistance, rigidité, élasticité

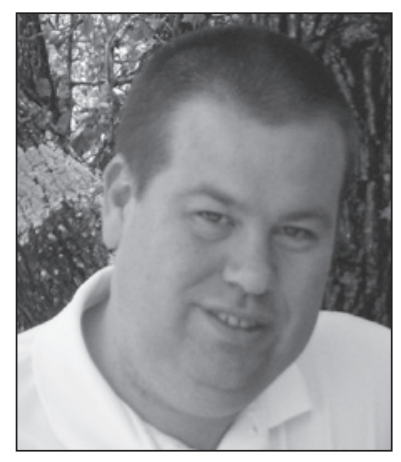

Brian K. Via

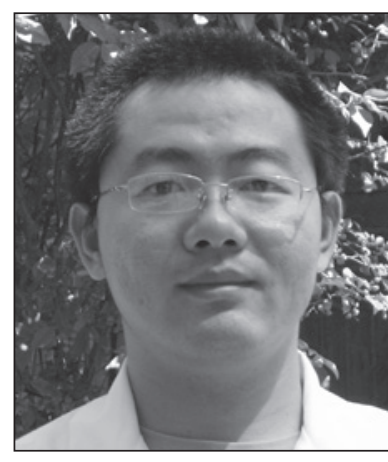

W. Jiang

\section{Introduction}

Wood quality in oriented strand board (OSB) manufacturing is often overlooked as a key source of variation in final product properties because manufacturers can adjust mat weight when feedstock quality declines. But increased mat weight costs manufacturers money and offers a competitive advantage to other regions or countries that utilized higher-density feedstocks. Additionally, having to adjust mat weight after the product has already failed to meet specification results in unnecessary quarantine and downgrade. Feed forward information about the direct mechanical properties of strands would thus be more accurate and minimize product downgrade.

Near-infrared reflectance (NIR) spectroscopy has become a popular technique to rapidly monitor the density and mechanical properties of solid wood increment cores, lumber, and composite products. NIR is sensitive to the fundamental bond vibrations, deformations, and stretches from key functional groups within the polymers in wood. When coupled with multivariate techniques, one can monitor the wood chemistry and related traits. NIR is a rapid and often non-destructive technique that can be utilized in manufacturing to rapidly determine material and product properties resulting in the ability

\footnotetext{
${ }^{1}$ School of Forestry and Wildlife Science, Auburn University, 3301 Forestry \& Wildlife Sciences Building, Auburn, Alabama 36849 USA. ${ }^{2}$ Forest Products Development Center, Auburn University, 520 Deval Drive, Auburn, Alabama 36849 USA.

*Corresponding author. E-Mail: bkv0003@auburn.edu
} 
to differentiate the feedstock by market value. For example, under many current distribution systems, lower-value stems that may be most appropriate for bioenergy may be mixed in with higher-value logs resulting in lost value (Ragauskas et al. 2006, Levin et al. 2011, Reeves 2012).

The sensitivity of NIR to feedstock variation can be affected by various factors including age, species, genetics, silviculture, and geography. Within a species, perhaps age effects are most dominant on the NIR spectra of southern pine (Pinus spp.) due to extreme differences in density between earlywood $\left(0.3 \mathrm{~g} / \mathrm{cm}^{3}\right)$ and latewood $\left(0.7 \mathrm{~g} / \mathrm{cm}^{3}\right)$ (Via 2010). An increase in material density can result in increased absorbance of light, particularly at densities greater than $0.6 \mathrm{~g} / \mathrm{cm}^{3}$ (Schimleck et al. 2001, Via et al. 2003, Via et al. 2012). The discrepancy between absorbance of light at low versus high density results in a nonlinear response between absorbance and density across many near-infrared wavelengths. The effect of genetic control or silvicultural response is also strong but more limited than age effects. NIR signals have been shown to be sensitive to various traits under genetic and silviculture control including lignin, cellulose, hemicellulose, extractives, microfibril angle, and density (Schimleck et al. 2002, Perez et al. 2007, Via et al. 2007, Isik et al. 2011). Via et al. (2005a) demonstrated that tracheid length could be predicted with NIR but as a function of age. In that study, when models were restricted to specific age classes, statistically different coefficients within the model were found at different ages suggesting that an NIR model computed at one age might not be adequate for use at another age. Since then, little has been done to address this calibration problem, which could be problematic for genetics-based studies, although Schimleck et al. (2005) did develop calibration models that were independent of age. The NIR spectrum itself has also been shown to vary with age due to potential differences in functional groups associated with underlying wood chemistry (Via et al. 2008). It has been shown that lignin and cellulose content within the woody cell wall will covary with microfibril angle resulting in an interactive effect on the modulus of rupture attributable to the plastic deformation within the lignin matrix at high microfibril angles and subsequent forces (Via et al. 2009). This may help to partially explain the ability of NIR to calibrate for microfibril angle and consequently modulus of rupture (MOR), both of which have been shown to be important traits measured through NIR for the forest products industry (So et al. 2004, Tsuchikawa 2007).

One important consideration for the calibration of NIR spectra to mechanical properties in tension has been the lack of standards for wood flakes. Testing strands in pure tension differs from current standards due to the size effect of strands, but loading rates and span distances for rectangular shaped specimens have been established (Han et al. 2006, Jeong et al. 2008). Utilization of NIR for pure tension has also been successful but with moderate correlation (Tsuchikawa et al. 2005). On the other hand, tension forces occur in bending and NIR has been shown to do an excellent job at predicting the modulus of elasticity (MOE) and MOR of wood (Via et al. 2003). As such, for monitoring flakes in a quality control setting, it may be better to focus on the development of calibrations for mechanical properties for flakes loaded in bending.

Another important consideration for the calibration of NIR spectra to wood mechanics is to determine if the assumption of model linearity is correct and if it is not, determine ways to account for this nonlinear behavior. Principal components regression (PCR) and partial least squares regression (PLS) are two common linear-based techniques. In the presence of nonlinearity between the spectra and the independent variable, often more linear-based factors can be utilized to account for the nonlinear effects. Another pre-treatment method would be to transform the data by taking the first derivative or other preprocessing methods. Application of the first derivative has been shown to partially offset the nonlinear effect of wood density on absorbance that occurs in the near-infrared region (Via et al. 2012). Conversely, application of the first derivative typically only resolves for small deviations from linearity. Two other techniques for handling nonlinear behavior are data splitting coupled with local linear modeling or nonlinear calibration techniques, which could include nonlinear higher order or interaction modeling with PCR, polynomial PLS, kernel regression methods, or neural networks (Centner et al. 2000, Mora and Schimleck 2010, Balabin et al. 2011, Via et al. 2012).

The objective of this study was to determine if nonlinear (higher order) principal component variables could improve the prediction of MOR and MOE for wood strands. Interpretations of the sources of nonlinearity were investigated. Wavelengths were assigned based on the literature and through NIR calibrations performed on laboratory wood chemistry performed in the lab.

\section{Methods \\ Materials}

Sawn strands were produced from small lumber segments of southern pine that were random in length and were obtained from a local truss plant. Nevertheless, each piece of lumber was classified as southern pine based on the grain, presence of large and numerous resin canals, and an abrupt transition from earlywood to latewood. A wide range of rings per inch and latewood percent were identified and included in the study to ensure a wide range in mechanical properties and to ensure a wide representation of trees. Several trips were made to the truss plant and significant time between sample selections were allowed to ensure that each piece of lumber came from a different tree. The lumber segments were reduced to $25 \mathrm{~mm} \times 25 \mathrm{~mm} \times 200 \mathrm{~mm}$ length clear samples and placed in a humidity chamber at $22^{\circ} \mathrm{C}$ and $50 \%$ relative humidity. The samples were allowed to equilibrate to a constant weight prior to testing.

These small clear samples were then ripped along the radial face to produce strands of approximately $200 \mathrm{~mm}$ length $\times 25$ $\mathrm{mm}$ width $\times 1.0 \mathrm{~mm}$ thickness and these sawn strands were returned to the humidity chamber and were reconditioned at $22^{\circ} \mathrm{C}$ and $50 \%$ relative humidity. A total of 51 sawn strands were manufactured for NIR scanning and mechanical testing.

\section{Near-infrared spectroscopy}

A Perkin Elmer Spectrum 400 FT-IR/FT-NIR Spectrometer was used to collect the NIR spectra. Absorbance was recorded from $1000 \mathrm{~nm}$ to $2500 \mathrm{~nm}$ at a resolution of $0.5 \mathrm{~nm}$. Spectra were collected at four equidistant locations from the center (square pattern) on each strand, with each spectrum composed of eight individual scans with a beam diameter of approximately $7 \mathrm{~mm}$. The four individual spectra for each strand were averaged using PerkinElmer Spectrum 6.2 software to produce one average spectrum for each wood strand. The first derivative pretreatment was applied to remove any baseline variations that might increase the noise within the data matrix. 


\section{Mechanical properties testing}

Bending tests were conducted on the wood strands using a Zwick-Roell load frame equipped with $10 \mathrm{kN}$ load cell and a computer-controlled screw-drive crosshead. Bending tests were conducted in three-point setup with a loading rate of $4.0 \mathrm{~mm} /$ $\mathrm{min}$, and support separation of $75 \mathrm{~mm}$.

\section{Wood chemistry determination}

Solid wood samples of southern pine were randomly selected from an inventory of southern pine lumber and were ground in a Wiley mill to 80 mesh. Wet chemistry analysis and highperformance liquid chromatography (HPLC) methods were performed to test extractives, lignin and monosaccharides content following the standard procedure developed by National Renewable Energy Laboratory (NREL) (Sluiter et al. 2008, 2012). The sugar content was determined through HPLC and the cellulose and hemicellulose contents were calculated assuming a galactose:glucose:mannose ratio of 1:1:3 in which cellulose is the sum total of glucose minus one-third of mannose and hemicellulose is the remaining amount for softwoods (Kitani and Hall 1989). When conducting wet chemistry, all samples were air-dried and tested for moisture content to calculate the dry weight of the original samples. All experiments were duplicated twice. Fig. 1 summarizes the procedure for wood chemistry determination.

\section{Chemometric modeling}

Principal components regression (PCR) models were created using SAS v.9.1 and the first derivative was obtained by calculating the slopes between adjacent points across the entire spectrum.

The same pre-treatment and data analysis techniques were used to compute and reduce the spectrum to $10 \mathrm{~nm}$ intervals, normalize each spectrum, compute the first derivative, and run regression and diagnostic analysis (Via 2010). The Mallows $\mathrm{Cp}$, RMSEP, RPD, and Press statistic were computed in accordance with the literature (Neter 1996, Maranan and Laborie 2008, Via 2010). Standard multiple linear regression methods were applied to the principal components $\left(\mathrm{p}_{\mathrm{i}}\right)$ to predict mechanical properties and Mallows $\mathrm{Cp}$ statistic was utilized to choose significant $\mathrm{p}_{\mathrm{i}}(\alpha=0.05)$. The complete wavelength range $(1000-2500 \mathrm{~nm})$ was utilized in PCR analysis.
Full model polynomials were computed through SAS 9.1 using the PROC REG procedure according to the following equation:

$$
\text { [1] } \quad \mathrm{y}=\mathrm{b}_{0}+\mathrm{b}_{1}{ }^{*} \mathrm{p}_{1}+\mathrm{b}_{2}{ }^{*} \mathrm{p}_{1}{ }^{2}+\mathrm{b}_{3}{ }^{*} \mathrm{p}_{1}{ }^{3}+\ldots+\mathrm{e}
$$

where $\mathrm{y}=$ mechanical property, $\mathrm{b}_{0}$ is the intercept, $\mathrm{b}_{\mathrm{i}}$ are the regression coefficients, $e$ is the error term, and $p_{i}$ are the principal components. The full model was then reduced to a smaller model based on the lowest Mallows Cp statistic that was tested for all possible model combinations. The number of principal components $\left(\mathrm{p}_{\mathrm{i}}\right)$ was limited to a maximum of 10 . The model cross validation as per the PRESS equation above was then used to compute RMSEP.

For wood chemistry determination with NIR, since interpretation and nonlinear modeling were not desirable, partial least squares regression methods were found to provide the best precision in the predicted value and were employed to predict lignin, cellulose, hemicellulose, and extractives. Several pre-treatments, such as multiplication scattering correction (MSC) and first derivative (FD), were selected to reduce the noise. Thirteen samples were used to construct the models and three samples were used to validate the models. The range of validation set was also inspected to ensure it was representative of the range of the calibration set. A summary of the wet chemistry coupled with NIR modeling can be seen in Fig. 1.

\section{Results and Discussion \\ Linear PCR}

Conventional multiple linear regression was applied with the independent variables being $p_{i}$ that were obtained from the near-infrared spectra. The $R^{2}$ for the MOE was much higher than MOR (Table 1). The better performance for MOE was not surprising since MOE is calculated from the slope of the stress strain curve, which avoids plastic deformation or fractures that can add variation to the data. The magnitude of difference in $R^{2}$ was somewhat unexpected since MOR has been shown to be well predicted by NIR-based models for solid wood (Via et al. 2003).

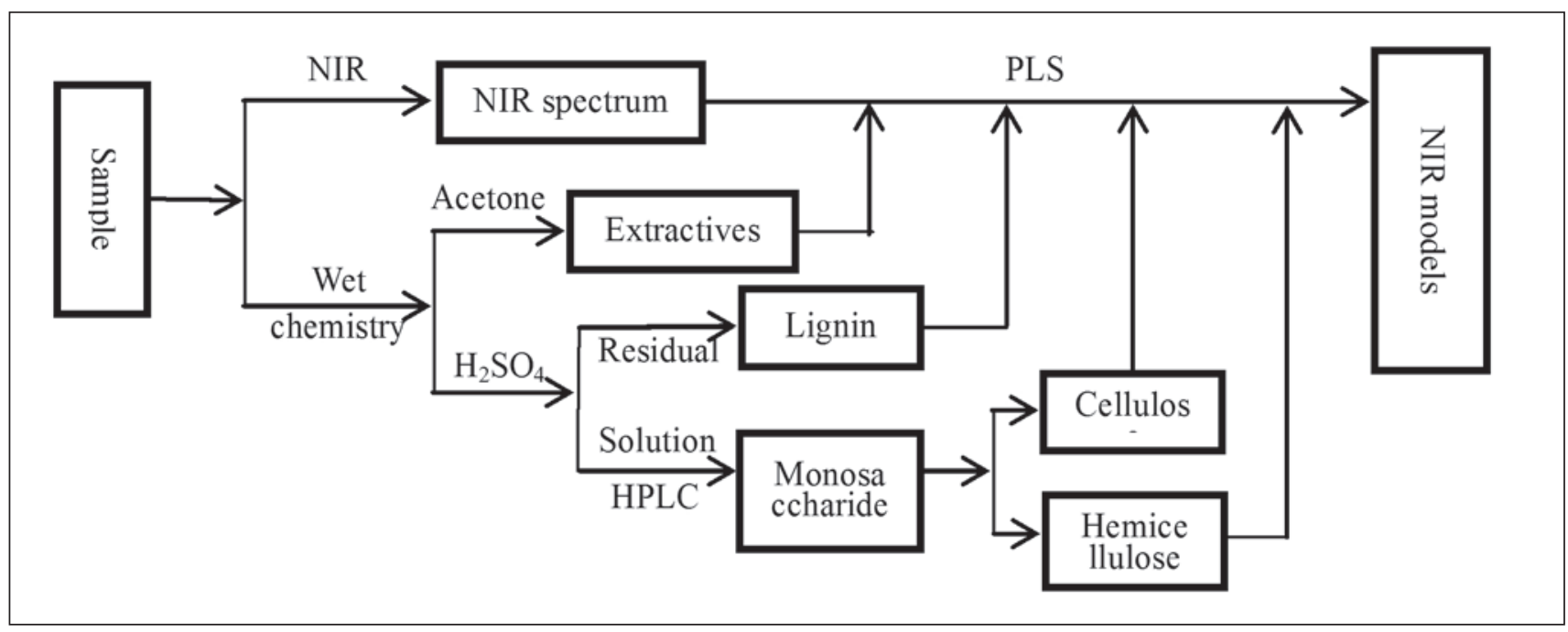

Fig. 1. Summary of wet chemistry procedure coupled with NIR modeling. 
Table 1. Calibration and full cross validation statistics for MOR and MOE using linear and non-linear models ( $N=51$ including leave one out cross validation)

\begin{tabular}{|c|c|c|c|c|c|c|c|}
\hline Model & Parameters $^{\mathrm{a}}$ & Cp & $\mathbf{R}^{2}$ & Adj. $R^{2}$ & RMSEC & RMSEP & $\mathbf{R P D}^{\mathrm{b}}$ \\
\hline Linear PCR MOE & $\begin{array}{l}\mathrm{p} 2, \mathrm{p} 4, \mathrm{p} 5 \\
\mathrm{p} 6, \mathrm{p} 7\end{array}$ & 7.8 & 0.76 & 0.73 & 613.3 & 663.7 & 1.8 \\
\hline $\begin{array}{l}\text { Nonlinear PCR } \\
\text { MOE }\end{array}$ & $\begin{array}{l}\text { p1 } \mathbf{1}^{3}, \mathrm{p} 2, \mathrm{p} 4 \\
\mathrm{p} 5, \mathbf{p} 5^{2}, \mathbf{p} 6^{3} \\
\mathrm{p} 7, \mathbf{p} 7^{2}, \mathbf{p} 7^{3}\end{array}$ & 6.6 & 0.83 & 0.79 & 543.6 & 664.0 & 1.8 \\
\hline Linear PCR MOR & $\mathrm{p} 2, \mathrm{p} 4$ & 5.3 & 0.45 & 0.41 & 12.3 & 13.3 & 1.2 \\
\hline $\begin{array}{l}\text { Nonlinear PCR } \\
\text { MOR }\end{array}$ & $\begin{array}{c}\mathrm{p} 2, \mathrm{p} 4, \mathrm{p} 5 \\
\mathrm{p} 5^{2}, \underset{\mathrm{p} 5^{3}, \mathrm{p} 7}{\mathrm{p} 7^{2}}\end{array}$ & 2.2 & 0.61 & 0.54 & 11.0 & 12.6 & 1.3 \\
\hline $\begin{array}{l}\text { Nonlinear PCR } \\
\text { first derivative } \\
\text { MOR }\end{array}$ & $\begin{array}{c}\mathrm{p} 2, \mathbf{p} 2^{2}, \mathbf{p}^{3}, \\
\mathrm{p} 3, \mathrm{p} 4, \mathrm{p} 7\end{array}$ & -1 & 0.61 & 0.55 & 10.9 & 12.6 & 1.3 \\
\hline
\end{tabular}

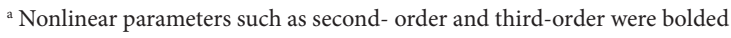

${ }^{\mathrm{b}}$ Standard deviation $=16.1 \mathrm{MPa}(\mathrm{MOR})$ and $1192 \mathrm{MPa}(\mathrm{MOE})$ was used for RPD computation which is the ratio of the reference to RMSEP.

Note: Polynomials usually follow the form of $M O R=b+a x^{3}+a x^{2}+a x^{3}+\ldots$ But if a reduced equation was determined to perform similarly with less terms than it was utilized for analysis and validated using the cross validation technique.

\section{Nonlinear PCR modeling}

Nonlinear models were also computed by testing if $\mathrm{p}_{\mathrm{i}}$ was significant for second- and third-order effects (Table 1). It can be seen that an additional $7 \%$ of the variation in MOE could be accounted for when nonlinear PCR analysis was performed. However, there were mixed results in terms of prediction capability. The RMSEP was still high when predicting MOE with nonlinear methods and this was coupled with high Cp values. A $\mathrm{Cp}$ value of no more than the number of parameters is generally desirable and indicative of a robust model. On the other hand, the adjusted $R^{2}$ did not drop off when predicting MOE, which is a sign of a robust model. These varied results suggest that better models might need to be employed for prediction of future populations, but it should be reasonable to use these models for interpretation purposes (which will be discussed later). Additionally, application of experimental designs during sample selection may also be beneficial during nonlinear calibration (Via et al. 2012).

The prediction of MOR through nonlinear PCR exhibited better results. Applying second- and third-order effects to parameters for MOR prediction improved the $\mathrm{R}^{2}$ dramatically. This was reflected in the RMSEP and Cp calculations and this resulted in less bias in residuals during prediction (data not shown). The conventional linear PCR model was perhaps not as robust since the Cp was not lower than the number of parameters in the model. More importantly, the increase in model robustness with nonlinear modeling supports the supposition that prediction of MOR with NIR spectra may be nonlinear in relation. This may be due to a) the nonlinear relationship between density and spectra (Via 2010, Via et al. 2012) b) the nonlinear covariance between wood chemistry, Fibre morphology, age, and spectra that is apparent (Via et al. 2007, 2009) and c) the nonlinear relationship between microfibril angle and lignin with higher polymer angles resulting in increased plastic deformation in lignin just prior to Fibre rupture, which results in an apparent nonlinear relationship between microfibril angle and MOR for southern pine and Douglas-fir (Pseudotsuga menziesii var. menziesii [Mirb.] Franco) (Via et al. 2009, Lachenbruch et al. 2010, Kohan et al. 2012).

Fig. 2 demonstrates the distribution of loadings (coefficients) across all wavelengths for $\mathrm{p}_{2}$, which was important in predicting MOR when second- and third-order effects were added. A normal distribution in loadings was found and therefore warranted
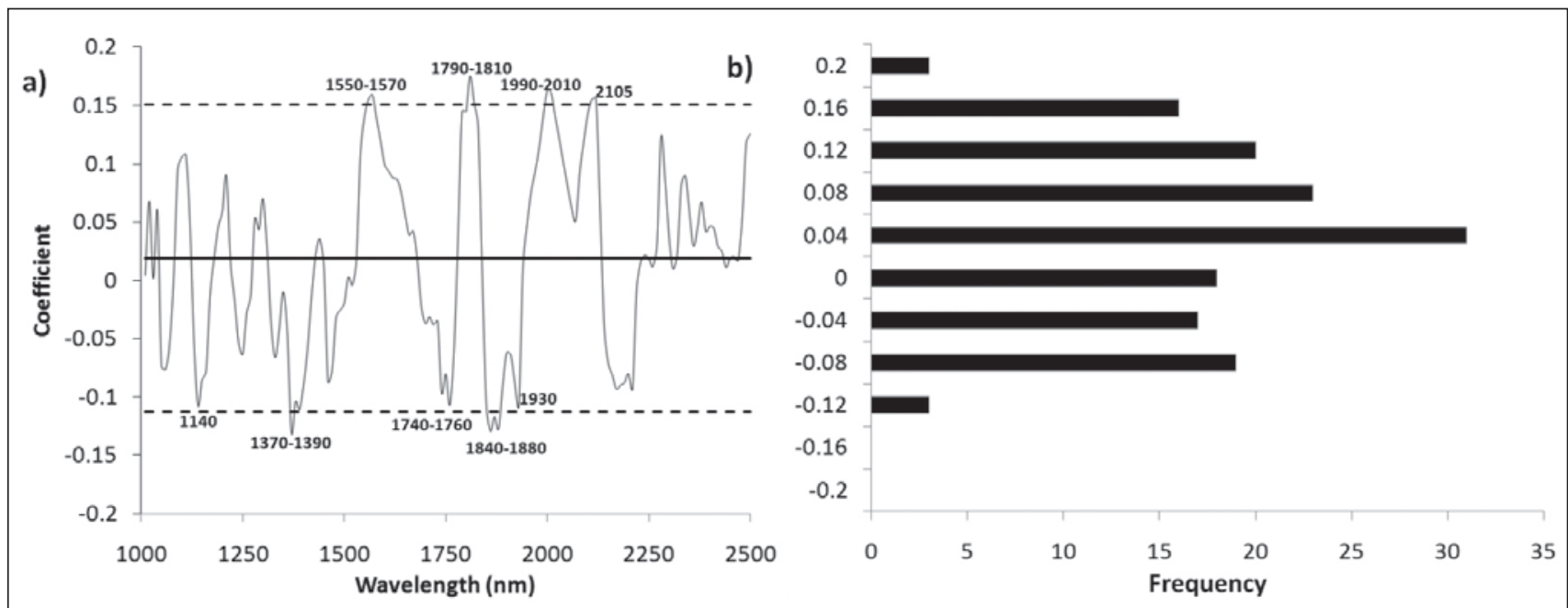

Fig. 2. Coefficients for principal component 2 which was statistically significant in the prediction of MOR as a third-order ( $p$-value $\leq 0.0001$ ) and second- order ( $p$-value $=0.0007$ ) parameter. 
the calculation of the lower and upper fifth percentile as represented by the dotted line. $\mathrm{P}_{2}$ was determined to be significant in the nonlinear prediction of MOR based on significant p-values while the loadings that exceeded the lower and upper fifth percentile were classified as being on the tail ends of the loading distribution and therefore were classified as the most important wavelengths in the prediction of MOR. Table 2 represents a summary of common wavelengths for significant $\mathrm{p}_{\mathrm{i}}$ that were useful in the prediction of MOR and MOE.

Nonlinearity between $\mathrm{p}_{\mathrm{i}}$, MOR, and MOE (Table 1) could be seen in studentized residual plots when conventional linear models were used (Fig. 3a and 3b). The nonlinear effect of $p_{2}$ on MOR was more drastic than the mild nonlinear effect of $\mathrm{p}_{7}$ on MOE. Therefore, nonlinear models were utilized to demonstrate the fit of the model to MOE and MOR data (Fig. 4). As can be seen, both MOE and MOR still deviate from linearity at high values despite the application of these nonlinear models but improvements in model statistics were observed (Table 1). This suggests that further variations in mechanical properties can be accounted for if better nonlinear models could be found. One method to improve the nonlinear model is to include interaction effects. Formal experimental designs must be employed to properly capture the interaction effect (Via et al. 2012).

Conversion of the spectra to the 1st derivative can be useful in further reducing the effect of nonlinearity between raw spectra and wood properties like density (Via et al. 2012). As such, the first derivative was applied to these data but without any real improvement in calibration statistics (Table 1). Because absorbance can deviate from a linear relationship to density, for high densities, the lack of improvement in the first derivative when predicting MOR (Table 1) and MOE (not shown) suggests that some other source of nonlinearity is unaccounted for in these calibration models.

\section{Band location}

Table 2. Assignment of key absorption bands to important wood chemistry constituents [Schwanninger et al. 2011]

wavelength $(\mathrm{nm}) \quad$ Wood chemistry association Bond vibration

\begin{tabular}{lll}
\hline 1143 & lignin & C-H stretch of $\mathrm{CH}_{3}$ groups \\
$1370-1390$ & hemicellulose & C-H stretch $+\mathrm{C}-\mathrm{H}$ deformation \\
$1550-1570$ & crystalline cellulose & O-H stretch \\
1810 & lignin & C-H stretch \\
$1930 ; 1980$ & water & O-H asymmetrical stretch and \\
2110 & cellulose & O-H deformation \\
2200 & lignin & O-H deformation + O-H stretch \\
& & C-H stretch + C=O stretch
\end{tabular}

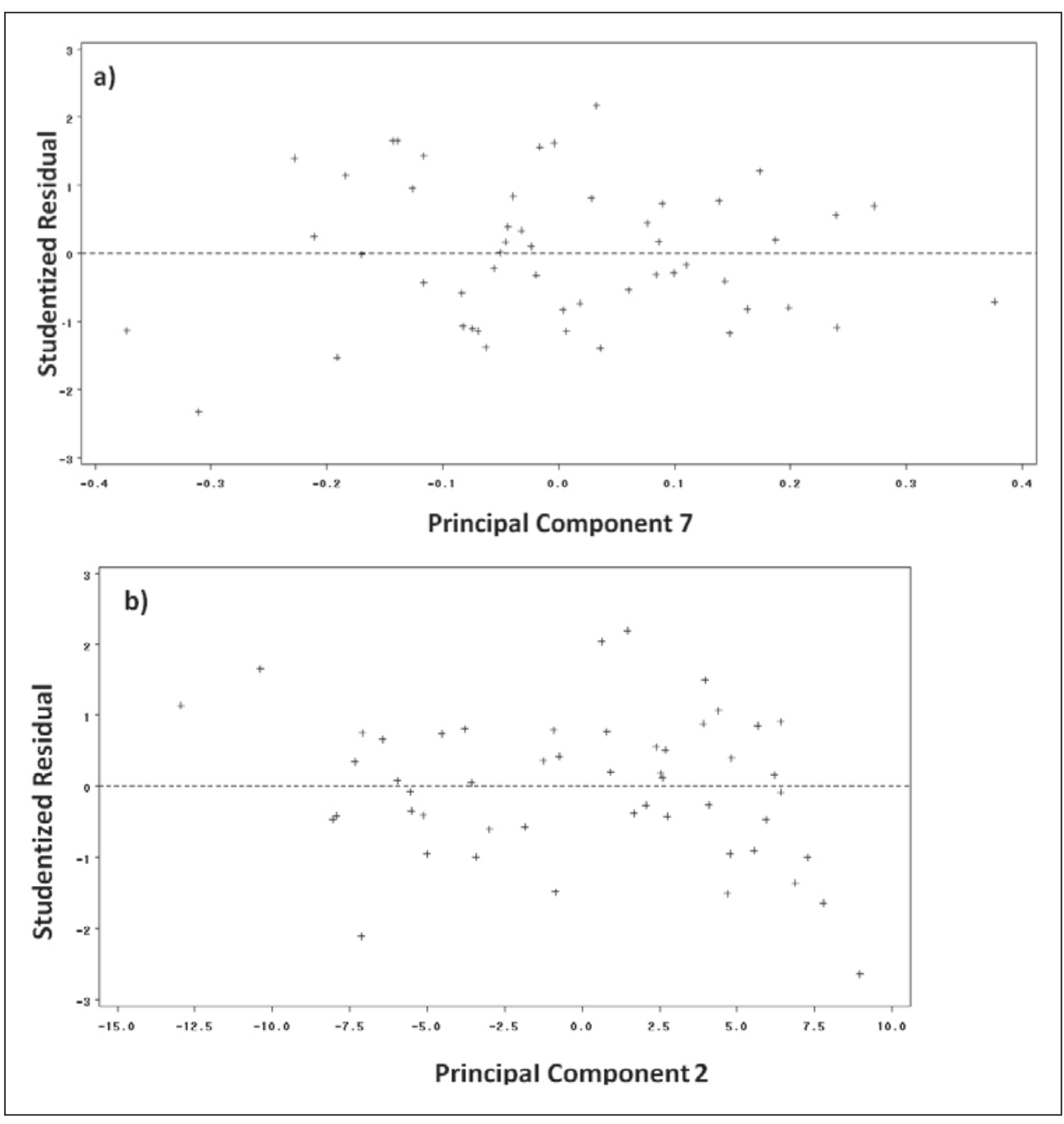

Fig. 3. Studentized residual values derived from linear principal components regression model for a) MOE and b] MOR for demonstration of nonlinearity tendency for specific Principal Components during multiple linear regression modeling.

The low RPD for the nonlinear (and linear) models suggests that at best these models can only be used to screen individual wood strands for strength and stiffness (Via 2013). However, perhaps that undermines the contribution that these models could make to manufacturers. Manufacturers would not care about the strength and stiffness of an individual strand but would instead like to monitor the population distribution 

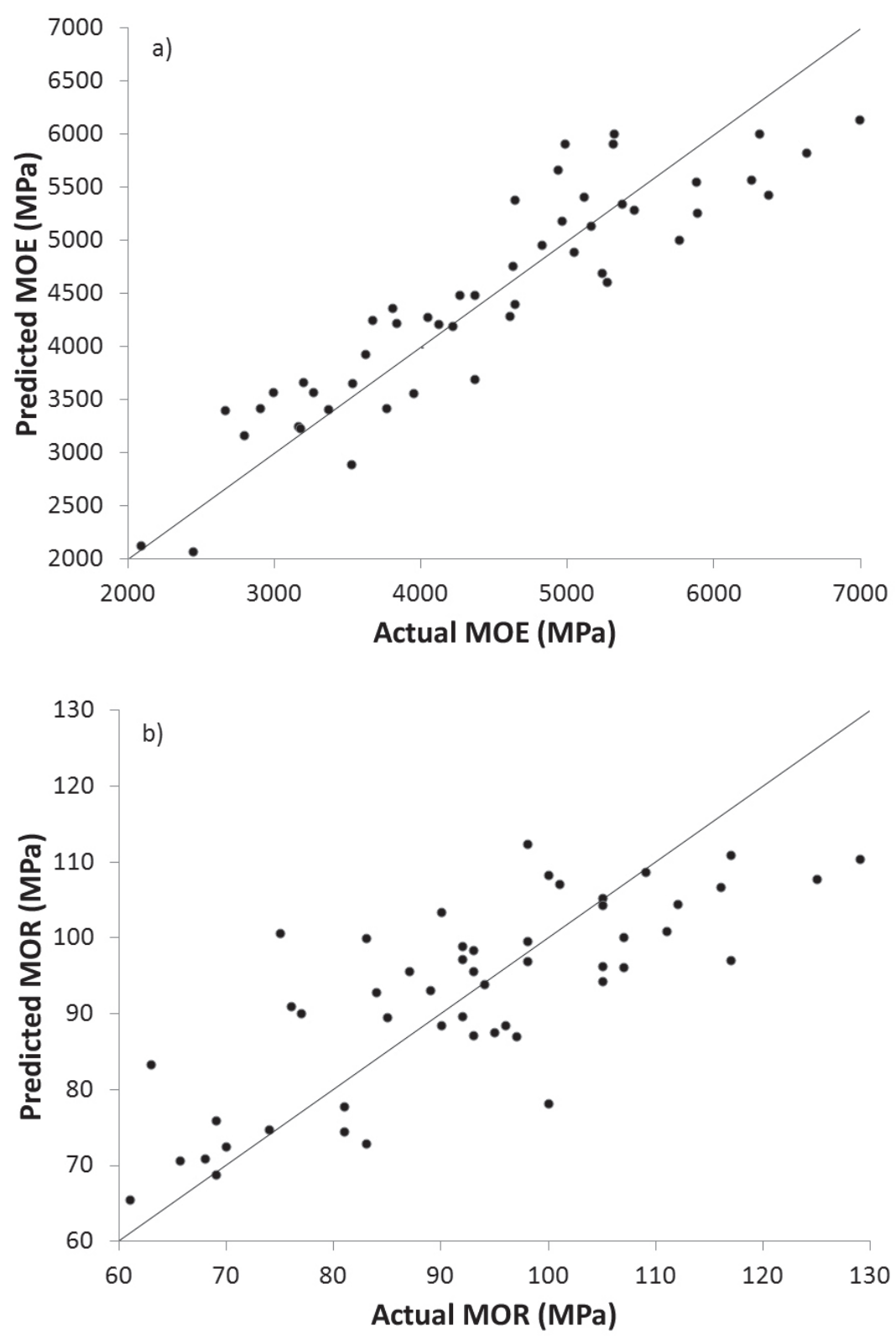

Fig. 4. Correlation between a) measured MOE and the optimal nonlinear model of MOE (after 1 st derivative pre-treatment) and b) measured MOR versus the optimal nonlinear model of MOR.

statistics. This has been done for density where the approximate RPD was low but measurement of 20 to 30 samples (for NIR predicted density) was shown to accurately reflect the populations average, variance, and distributional shape (Via et al. 2005b). The maximum load for oriented strand board has also been modeled from NIR spectra and similar recommendations were made (Via et al. 2012).

\section{Interpretation of models}

Fig. 5 demonstrates the nonlinear relationship between $p_{2}$ and MOR. The parameters in the model were chosen based on p-values (Table 3 ) and $p_{2}$ and $p_{7}$ were the two parameters to exhibit a nonlinear relationship with MOR. For $\mathrm{p}_{2}$, lignin played the largest role but cellulose, water, and hemicellulose associated wavelengths were also important. The strong importance of lignin on MOR was expected since lignin experiences plastic deformation prior to rupture (Via et al. 2009). Nevertheless, the $1560 \mathrm{~nm}$ and $2110 \mathrm{~nm}$ were attributable to the crystalline and bulk cellulose polymer respectively. Furthermore, water also played a role with increased moisture resulting in lower mechanical properties. The role of water was not surprising for it is well known that variation in moisture content affects the rupture properties of wood. However, it was interesting in this case because these flakes were conditioned to a constant relative humidity and temperature and were supposed to be consistent in moisture content during testing. It was hypothesized that the importance of water, despite strand conditioning, may be attributable to differences in drying history between strands in which different moisture contents are possible at the same relative humidity and temperature due to differences in adsorption and desorption histories for each strand. It has been shown that absorption and desorption for the same piece of wood results in different moisture contents and that NIR is sensitive to these differences (Inagaki et al. 2008). Furthermore, despite attempts to control room temperature during NIR scanning, these samples were thin and can quickly reach a new equilibrium upon exposure to a different relative humidity and temperature. Because NIR is very sensitive to moisture changes (Via et al. 2013), it is possible that subtle changes in strand moisture could have added some error to the calibration equation. For industrial applications, the moisture is not laboratory-controlled and suggests that models herein may be slightly optimistic or more factors for prediction may be necessary to partition out addition environmental variation.

The response in MOE to principal components was also nonlinear but not as significant as when MOR was modeled (Table 1). This was probably attributable to a baseline shift in spectra to density (which was observed in this data) for most wavelengths coupled with the strong relationship between density and MOE (Via et al. 2010, Lachenbruch et al. 2010).

\section{Validation of wavelengths attributable to wood chemistry}

It should be noted that to this point, the assignment of key wavelengths to underlying wood chemistry was done through the literature (Schwanninger et al. 2011). However, this study went one step further by calibrating the NIR to wood chemistry 


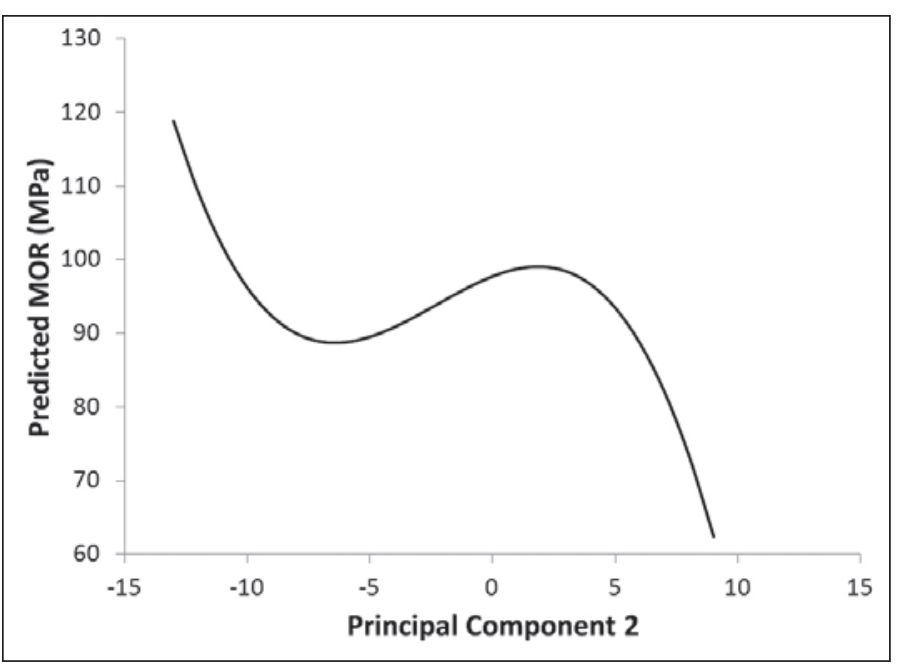

Fig. 5. Nonlinear plot of predicted MOR as a function of $\mathrm{p} 2, \mathrm{p}^{2}$, and $\mathrm{p}^{3}$. Other linear parameters (p3, p4, and p7) were kept constant and the range from the actual data was used for the x-axis. content and the loadings within the models were used to determine key wavelengths that were important to wood chemistry and consequently wood mechanical properties. Table 4 demonstrates the ability of the constructed models to predict lignin, extractives, cellulose, and hemicellulose. NIR was most sensitive to lignin and cellulose but was still significantly capable at modeling hemicellulose and extractives (Table 4). Fig. 6 demonstrates the validation of NIR to wet chemistry analysis. These data generally fell on the 1:1 line for validation data and this was indicative of good model accuracy.

The wavelengths that exhibited high loadings for the prediction of lignin were $1060,1355,1385,1422,1688$, 1905, 1947, 2037, 2144, 2272, 2358, and $2431 \mathrm{~nm}$ (Fig. 7a). Of these wavelengths, half were similar to wavelengths that were important in prediction of strand mechanical properties: 1380, 1680, 1910, 1950, 2271, and $2352 \mathrm{~nm}$. It is important to note that not all of these wavelengths were attributable to lignin as indicated in Table 2 and 3. For example, 1910 was possibly attributable to hemicellulose or water due to $\mathrm{C}=\mathrm{O}$ stretch in hemicellulose or $\mathrm{O}-\mathrm{H}$ asymmetric stretch in water. The reason for other

Table 3. Statistically important principal components, wavelengths, and assigned chemistry for the prediction of MOE and MOR

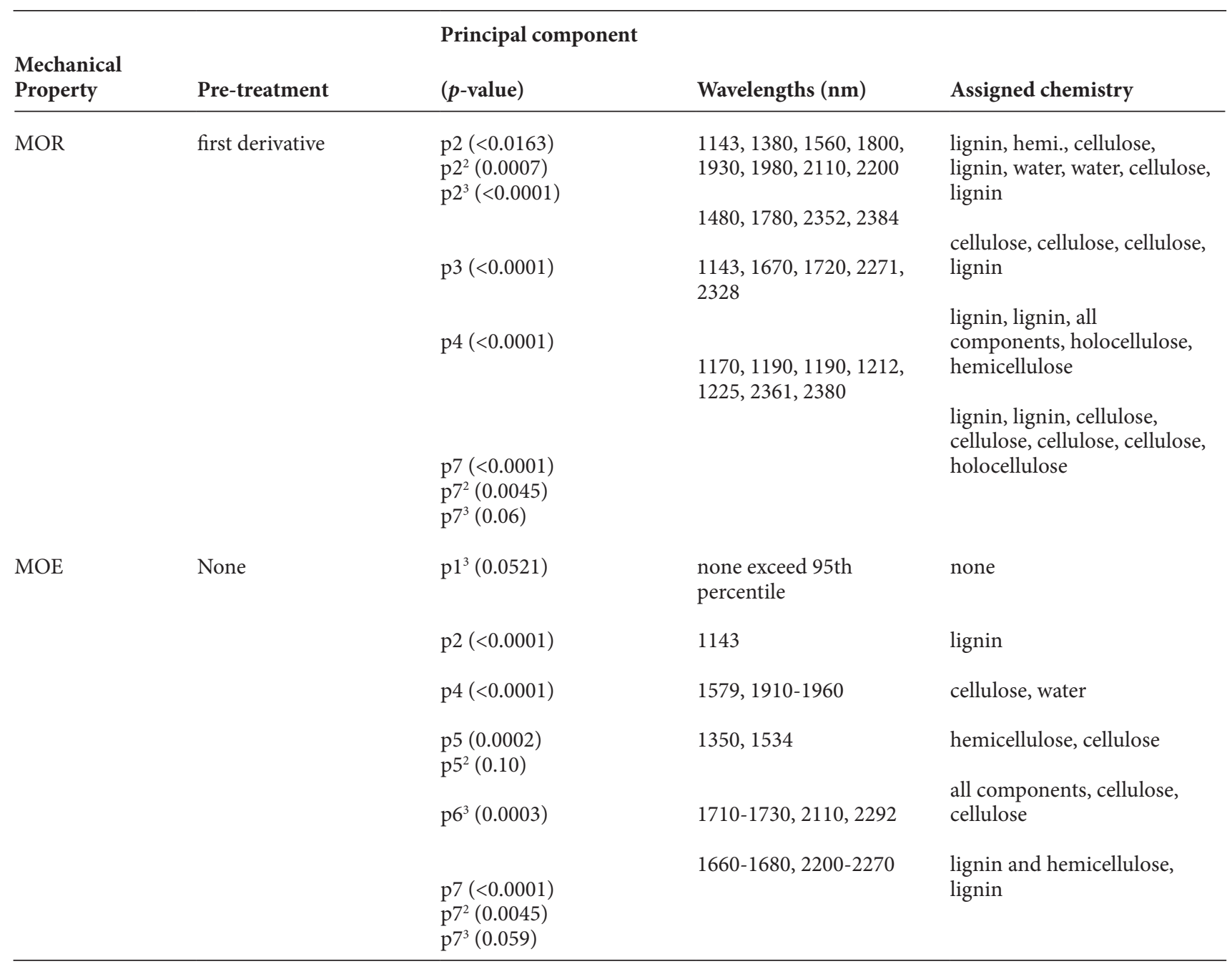

Note: Wavelengths were listed as important if the eigenvector loadings for the principal component exceeded two standard deviations from the mean loading. 


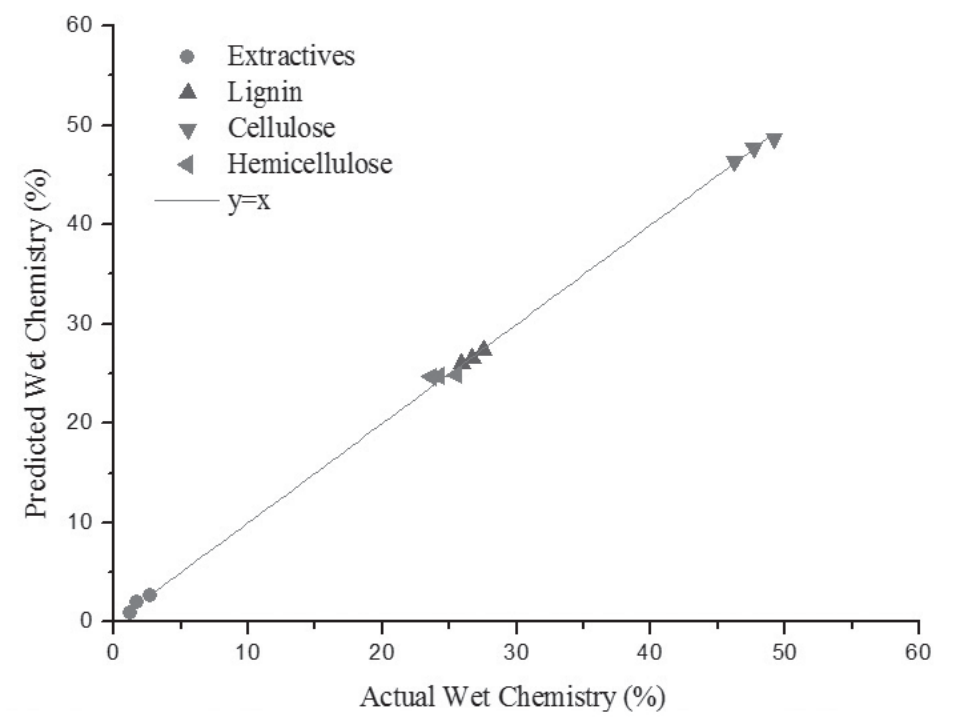

Fig. 6. Validation of actual wood chemistry NIR models on new samples that were not included in the calibration data set. wood polymers playing a role in the prediction of lignin could be due to the fact that we were measuring composition percentages. So for example, if lignin decreases, than either hemicellulose and/ or cellulose had to increase. Likewise, the reason that water associated wavelengths may be important is that lignin is a hydrophobic polymer and so reductions in lignin should result in changes in moisture content which in turn will influence mechanical properties.

The wavelengths most associated with cellulose were 1030,1145, 1201, 1286, 1481, 1578, 2052, 2218, and $2327 \mathrm{~nm}$ (Fig. 7b). This coincided with the following wavelengths that were important in prediction of mechanical properties: 1143, 1212, 1480, 1579, 2218, and $2328 \mathrm{~nm}$. Six of nine wavelengths that were associated with the prediction of cellulose were also important in the prediction of wood mechanical properties. As with the prediction of lignin, not all wavelengths that were important in the prediction of cellulose were actually attributable to cellulose (Table 3 ).

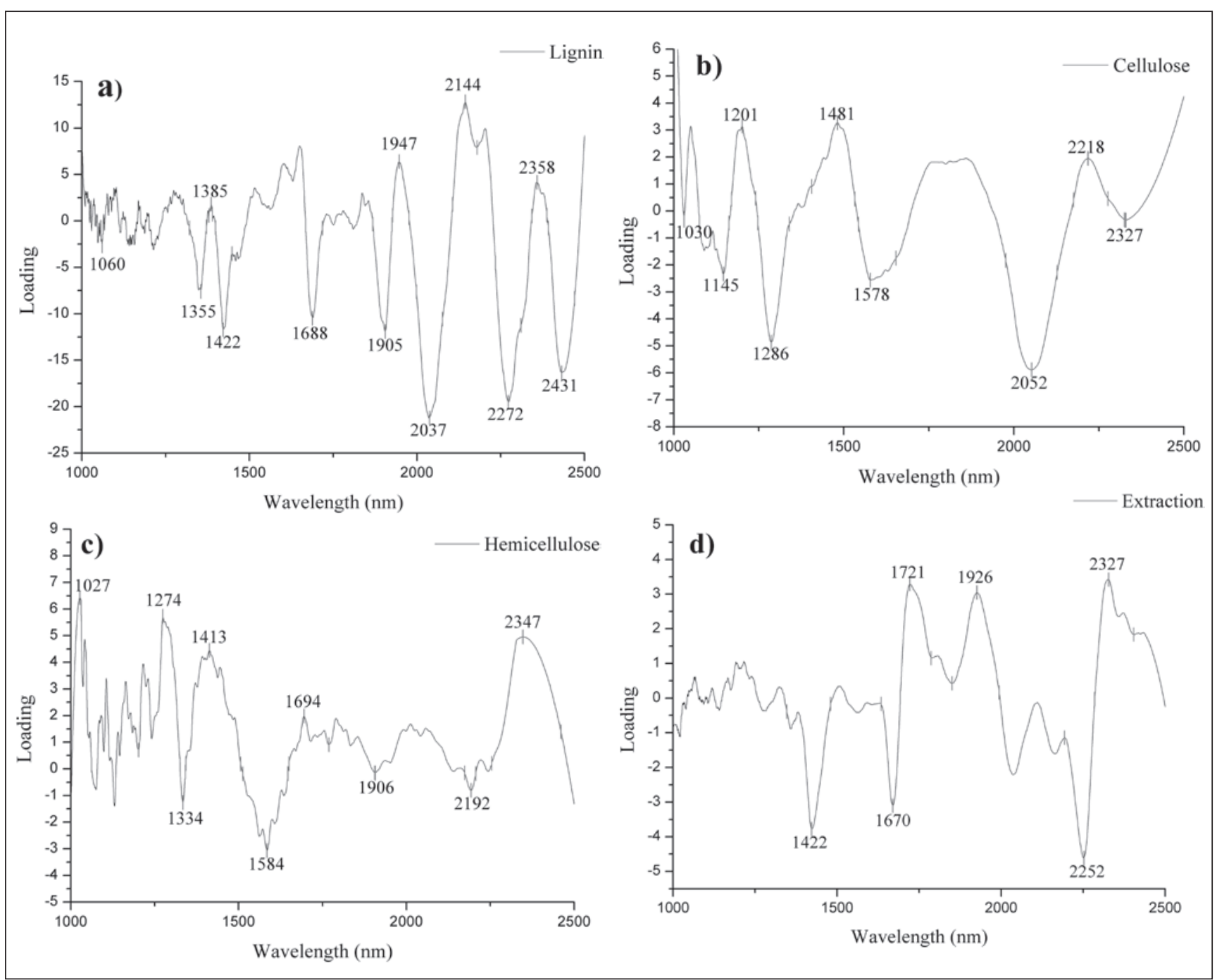

Fig. 7. PLS-derived loadings for calibration curves for a) lignin b) cellulose c) hemicellulose and d) extractives. 
Table 4. Calibration and validation statistics for wood chemistry $(N=13$ for calibration and 3 for validation]

\begin{tabular}{lcccccc}
\hline Model & Parameters & $\mathbf{R}^{2}$ & Adj. $\boldsymbol{R}^{2}$ & RMSEC & RMSEP & RPD \\
\hline Linear PLS - lignin & 3 & 0.99 & 0.98 & 0.13 & $0.15^{\mathrm{a}}$ & 9.87 \\
Linear PLS - extractives & 3 & 0.81 & 0.77 & 0.38 & $0.25^{\mathrm{b}}$ & 2.98 \\
Linear PLS - cellulose & 3 & 0.96 & 0.95 & 0.43 & 0.35 & 4.23 \\
$\begin{array}{l}\text { Linear PLS - } \\
\text { hemicellulose }\end{array}$ & 3 & 0.87 & 0.84 & 0.48 & 0.76 & 1.26 \\
\hline
\end{tabular}

a Note that the RMSEP was much lower than the standard deviation of replicates for the same lignin sample which was approximately 0.54 for a sample size of 16 .

b The RMSEC was calculated based on 13 samples while the RMSEP was calculated based on 3 samples and the low samples size of 3 resulted in higher error in parameter estimation resulting in RMSEP $<$ RMSEC, which would have not likely occurred for higher samples sizes.

\section{Acknowledgements}

This research was funded by the Hatch Formula Grant provided by the Alabama Agricultural Experiment Station. Mcintire-Stennis support is also acknowledged in which the goal was to use spectroscopic modeling during the development of wood composites. The wood chemistry was supported by grants obtained from the Department of Energy and Agriculture and Food Research Initiative (AFRI) CAP. Finally, the Forest Products Development Center is acknowledged for support of this project.
The wavelengths $1143 \mathrm{~nm}$ and $2328 \mathrm{~nm}$ were attributable to lignin and hemicellulose respectively; however, they did exert the least influence in the prediction of cellulose as indicated by the magnitude of the loadings in Fig. $7 \mathrm{~b}$.

The wavelengths most associated with hemicellulose were $1027,1274,1334,1413,1584,1694$, and $2347 \mathrm{~nm}$. This coincided with the following wavelengths that were important in the prediction of mechanical properties: 1350, 1694, and $2352 \mathrm{~nm}$. Only $2352 \mathrm{~nm}$ was not attributable to hemicellulose but instead due to the C-H stretch and deformation in cellulose (Schwanninger et al. 2011).

Extractives were not anticipated to affect the mechanical properties since they do not provide any structural support for the tree. However, they do have phenolic compounds that can rival lignin resulting in additional noise to the spectra and was thus measured for completeness. The wavelengths most associated with extractives were 1422, 1670, 1721, 1926, 2252, and $2327 \mathrm{~nm}$. This coincided with the following wavelengths that were important in the prediction of mechanical properties: 1670 and $1926 \mathrm{~nm}$. The overlap between extractives and lignin at $1670 \mathrm{~nm}$ was probably attributable to the similarity in phenolic structures that are present in both. This similarity between lignin and extractives suggests that removal of wood extractives prior to calibration may improve model robustness. The wavelength $1926 \mathrm{~nm}$ was attributable to water and may vary as a function of water content since extractives can act as a bulking agent within the wood, resulting in less sites for moisture sorption for wood with higher extractives contents (Nzokou and Kamdem 2004).

\section{Conclusions}

Near-infrared calibration models were developed for MOR and MOE for 51 samples that were prepared in similar dimension to strands that could be obtained from a conventional OSB plant. It was found that the percentage of variation in mechanical properties could be better accounted for when second- and third-order terms were added to the model. Interpretation of the independent variables identified the wood chemistry polymers that were responsible for the prediction of MOR and MOE. Models for wood chemistry were also developed and compared to mechanical property models. This comparison helped to confirm the underlying association between wood chemistry and mechanical properties that is suggested in the literature.

\section{References}

Balabin, R.M., E.I. Lomakina and R.Z. Safieva. 2011. Neural network (ANN) approach to biodiesel analysis: Analysis of biodiesel density, kinematic viscosity, methanol and water contents using near infrared (NIR) spectroscopy. Fuel 90(5): 2007-2015.

Centner, V., J. Verdu-Andres, B. Walczak, D. Jouan-Rimbaud, F. Despagne, L. Pasti, R. Poppi, D.L. Massart and O.E. de Noord. 2000. Comparison of multivariate calibration techniques applied to experimental NIR data sets. Appl. Spectrosc. 54(4): 608-623.

Han, G.P., Q.L. Wu and J.Z. Lu. 2006. Selected properties of wood strand and oriented strandboard from small-diameter southern pine trees. Wood and Fiber Science 38(4): 621-632.

Inagaki, T., H. Yonenobu and S. Tsuchikawa. 2008. Near-infrared spectroscopic monitoring of the water adsorption/desorption process in modern and archaeological wood. Appl. Spectrosc. 62 (8): 860-865.

Isik, F., C.R. Mora and L.R. Schimleck. 2011. Genetic variation in Pinus taeda wood properties predicted using non-destructive techniques. Ann. For. Sci. 68(2): 283-293.

Jeong, G.Y., D.P. Hindman, D. Finkenbinder, J.N. Lee and Z.Y. Lin. 2008. Effect of loading rate and thickness on the tensile properties of wood strands. Forest Prod. J. 58(10): 33-37.

Kitani, O. and C.W. Hall. 1989. Biomass handbook. pp. 135-136. Gordon and Breach Science Publishers, New York.

Kohan, N., B.K. Via and S. Taylor. 2012. Prediction of strand feedstock mechanical properties with near infrared spectroscopy. Bioresources 7(3): 2996-3007.

Lachenbruch, B., G.R. Johnson, G.M. Downes and R. Evans. 2010. Relationships of density, microfibril angle, and sound velocity with stiffness and strength in mature wood of Douglas-fir. Can. J. Forest Res. 40(1): 55-64.

Levin, R., S. Krigstin and S. Wetzel. 2011. Biomass availability in eastern Ontario for bioenergy and wood pellet initiatives. For. Chron. 87(1): 33-41.

Maranan, M.C. and M.P.G. Laborie. 2008. Rapid prediction of the chemical traits of hybrid poplar with near infrared spectroscopy. J. Biobased Mater. Bio. 2(1): 57-63.

Mora, C.R. and L.R. Schimleck. 2010. Kernel regression methods for the prediction of wood properties of Pinus taeda using near infrared spectroscopy. Wood Sci. Technol. 44(4): 561-578.

Neter, J. 1996. Applied linear regression models. 3rd ed. pp. 1-1408. Irwin, Chicago, IL.

Nzokou, P. and D.P. Kamdem. 2004. Influence of wood extractives on moisture sorption and wettability of red oak (Quercus rubra), black cherry (Prunus serotina), and red pine (Pinus resinosa). Wood and Fiber Science 36(4): 483-492. 
Perez, D.D., A. Guillemain, P. Alazard, C. Plomion, P. Rozenberg, J.C. Rodrigues, A. Alves and G. Chantre. 2007. Improvement of Pinus pinaster Ait elite trees selection by combining near infrared spectroscopy and genetic tools. Holzforschung 61(6): 611-622.

Ragauskas, A.J. et al. 2006. The path forward for biofuels and biomaterials. Science 311(5760): 484-489.

Reeves, J.B. 2012. Potential of Near- and Mid-infrared Spectroscopy in Biofuel Production. Commun. Soil Sci. Plan. 43(1-2): 478-495.

Schimleck, L., R. Evans and J. Ilic. 2001. Application of near infrared spectroscopy to a diverse range of species demonstrating wide density and stiffness variation. IAWA J. 22(4): 415-429.

Schimleck, L.R., R. Evans and A.C. Matheson. 2002. Estimation of Pinus radiata D. Don clear wood properties by near-infrared spectroscopy. J. Wood Science 48(2): 132-137.

Schimleck, L.R., P.D. Jones, G.F. Peter, R.F. Daniels and A. Clark. 2005. Success in using near infrared spectroscopy to estimate wood properties of Pinus taeda radial strips not due to autocorrelation. J. Near Infrared Spectroscopy 13(1): 47-51.

Schwanninger, M., J.C. Rodrigues and K. Fackler. 2011. A review of band assignments in near infrared spectra of wood and wood components. J. Near Infrared Spectroscopy 19(5): 287-308.

Sluiter, A., B. Hames, R. Ruiz, C. Scarlata, J. Sluiter, D. Templeton and D. Crocker. 2012. Determination of structural carbohydrates and lignin in biomass. NREL/TP-510-42618.

Sluiter, A., R. Ruiz, C. Scarlata, J. Sluiter and D. Templeton. 2008. Determination of Extractives in Biomass. NREL/TP-510-41619.

So, C-L., B.K. Via, L.H. Groom, L.R. Schimleck, T.F. Shupe, S.S. Kelley and T.G. Rials. 2004. Near infrared spectroscopy in the forest products industry. Forest Prod. J. 54(3): 6-16.

Tsuchikawa, S. 2007. A review of recent near infrared research for wood and paper. Appl. Spectrosc. Rev. 42(1): 43-71.

Tsuchikawa, S., Y. Hirashima, Y. Sasaki and K. Ando. 2005. Nearinfrared spectroscopic study of the physical and mechanical properties of wood with meso- and micro-scale anatomical observation. Appl. Spectrosc. 59(1): 86-93.
Via, B.K. 2010. Prediction of oriented strand board wood strand density by near infrared and Fourier transform infrared reflectance spectroscopy. J. Near Infrared Spectroscopy 18(6): 491-498.

Via, B.K. 2013. Characterization and evaluation of wood strand composite load capacity with near infrared spectroscopy. Mater. Struct. doi:10.1617/s11527-013-0018-4.

Via, B.K., S. Adhikari and S. Taylor. 2013. Modeling for proximate analysis and heating value of torrefied biomass with vibration spectroscopy. Bioresource Technol. 133: 1-8.

Via, B.K., T. McDonald and J. Fulton. 2012. Nonlinear multivariate modeling of strand density from near-infrared spectra. Wood Sci. Technol. 46(6): 1073-1084.

Via, B.K., T.F. Shupe, L.H. Groom, M. Stine and C-L. So. 2003. Multivariate modelling of density, strength and stiffness from near infrared spectra for mature, juvenile and pith wood of longleaf pine (Pinus palustris). J. Near Infrared Spectroscopy 11(5): 365-378.

Via, B.K., T.F. Shupe, M. Stine, C-L. So and L.H. Groom. 2005a. Tracheid length prediction in Pinus palustris by means of near infrared spectroscopy: the influence of age. Holz Als Roh-Und Werkstoff 63(3): 231-236.

Via, B.K., C.L. So, L.G. Eckhardt, T.F. Shupe, L.H. Groom and M. Stine. 2008. Response of near infrared diffuse reflectance spectra to blue stain and wood age. J. Near Infrared Spectroscopy 16(1): 71-74.

Via, B.K., C-L. So, L.H. Groom, T.F. Shupe, M. Stine and J. Wikaira. 2007. Within tree variation of lignin, extractives, and microfibril angle coupled with the theoretical and near infrared modeling of microfibril angle. IAWA J. 28(2): 189-209.

Via, B.K., C-L. So, T.F. Shupe, L.H. Groom and J. Wikaira. 2009. Mechanical response of longleaf pine to variation in microfibril angle, chemistry associated wavelengths, density, and radial position. Compos. Part A: Appl. S. and Man. 40(1): 60-66.

Via, B.K., C-L. So, T.F. Shupe, M. Stine and L.H. Groom. 2005b. Ability of near infrared spectroscopy to monitor air-dry density distribution and variation of wood. Wood and Fiber Science 37(3): 394-402. 